\title{
LA ENERgía RENOVABle en Argentina COMO ESTRATEGIA DE POLÍTICA ENERGÉTICA E INDUSTRIAL
}

\author{
María Eugenia Castelao Caruana ${ }^{a}$
}

Fecha de recepción: 23 de abril de 2018. Fecha de aceptación: 22 de octubre de 2018.

http://dx.doi.org/10.22201/iiec.20078951e.2019.197.64625

Resumen. Con la promulgación de un nuevo régimen sobre fomento a la energía eléctrica de fuentes renovables (2015), Argentina retomó la transición hacia una matriz energética más sustentable y diversificada. Desde entonces, la política energética nacional ha impulsado la creación de un mercado doméstico de Energía de Fuentes Renovables (EFR) competitivo, mientras que la política industrial se ha concentrado en promover la transferencia de tecnología y la industria local. El presente trabajo analiza la orientación de estas políticas, la coherencia de sus objetivos y los resultados inmediatos. La política energética y la política industrial destinada a la EFR se desarrollan a diferentes velocidades, con escasa coordinación, condicionando la participación de la industria nacional en sus cadenas de valor.

Palabras clave: energía renovable; energía eléctrica; política energética; fuentes renovables; política industrial; tecnología.

Clasificación JEL: O13; P28; Q42; Q43; Q48.

\section{Renewable Energy in Argentina as an Energy and Industrial Policy Strategy}

\begin{abstract}
With the enactment of a new regime regarding the promotion of electrical energy from renewable sources in 2015, Argentina took up the transition towards a more sustainable and diversified energy matrix. Since then, national energy policy has driven the creation of a competitive domestic market for Energy from Renewable Sources (ERS). Meanwhile, industrial policy has focused on promoting the transfer of technology and local industry. This paper analyzes the orientation of these policies, the coherence of their objectives, and the immediate results. Energy policy and industrial policies designed for ERs develop at different speeds and with little coordination, making it necessary for national industry to participate in its value chains.
\end{abstract}

Key Words: renewable energy; electrical energy; energy policy; renewable sources; industrial policy; technology.

\footnotetext{
${ }^{a}$ Centro de Estudios Urbanos y Regionales, Consejo Nacional de Investigaciones Científicas y Técnicas (CEUR-CONICET), Argentina. Correo electrónico: eugeniacastelao@conicet.gov.ar
} 


\section{INTRODUCCIÓN}

A inicios de la década de los noventa, diversos países adoptaron el compromiso de combatir el cambio climático mediante la transición hacia sistemas de energía más sustentables (Lund, 2009). Frente a la volatilidad de los mercados de combustibles fósiles, algunos países se propusieron mejorar la seguridad y flexibilidad de sus sistemas energéticos incorporando Energía de Fuentes Renovables (EFR). América Latina y el Caribe (ALC) enfrentan el desafío de integrar tecnologías de EFR para disminuir la dependencia de la matriz energética del petróleo (45.5\%), el gas natural (21.3\%) y la energía hidroeléctrica (23.2\%) (véase cuadro 1) y, por ende, mitigar su exposición a la variabilidad de los precios de los combustibles fósiles y el impacto del cambio climático en las reservas de agua (Jacobs et al., 2013). Asimismo, la región debe incrementar la producción de energía para responder al crecimiento de la demanda que, en el caso de la electricidad, se estima en una tasa anual de 1.6-1.9\% entre 2014 y 2030, y de $0.8-1.1 \%$ de 2030 a 2060 (World Energy Council, 2017).

Durante muchos años, las condiciones institucionales y tecnológicas que acompañaron el desarrollo de los mercados energéticos dieron lugar a mecanismos de lock-in ${ }^{1}$ que inhibieron el desarrollo y la difusión de las tecnologías asociadas a la EFR (Unruh, 2000; Frantzeskaki y Loorbach, 2010). Los efectos de aprendizaje, las economías de escala y alcance, las externalidades en red, la interrelación tecnológica, y las configuraciones sociotécnicas otorgaron ventajas a las tecnologías energéticas tradicionales, a pesar de sus impactos ambientales negativos (Cecere et al., 2014).

En la última década, las políticas de fomento a la EFR han logrado romper la inercia tecnológica de los mercados energéticos, impulsando la creación de mercados nacionales de EFR y promoviendo la competitividad y alcance de estas tecnologías (IRENA, 2017a). Este proceso se refleja en la creciente participación de estas tecnologías en la matriz energética de Alemania (13.4\%), España (11.3\%), Reino Unido (11\%), Chile (7.9\%) y Brasil (7.5\%) (véase cuadro 1). Impulsada por estos países, Europa encabeza la transición energética con una participación promedio de la EFR de 8\%, mientras que en ALC este

1 Se trata de mecanismos que favorecen la adopción de una tecnología y le otorgan ventajas sobre tecnologías alternativas, aun cuando estas últimas sean sustitutos potencialmente superiores. Por lo general, producen rendimientos crecientes a escala a medida que la tecnología se difunde, contribuyendo a que se torne dominante y marcando su sendero de innovación (Brian Arthur, 1989). Son el resultado de un proceso de coevolución entre la infraestructura tecnológica, las organizaciones, la sociedad y las instituciones de gobierno, que facilita la continuidad de una determinada tecnología (Unruh, 2002). 
porcentaje alcanza 4.7\%, cercano a la media de Estados Unidos de América y Canadá, pero por encima del resto de las demás regiones. Los datos ponen en evidencia las oportunidades de crecimiento que tiene la EFR a nivel mundial, pero también muestran que existe una fuerte asimetría entre países al interior de las regiones.

Cuadro 1. Matriz energética primaria: distribución del consumo por fuente de energía y país o región. En porcentaie (2017)

\begin{tabular}{|c|c|c|c|c|c|c|}
\hline País/Región & Petróleo & $\begin{array}{c}\text { Gas } \\
\text { natural }\end{array}$ & Carbón & $\begin{array}{l}\text { Energía } \\
\text { nuclear }\end{array}$ & $\begin{array}{l}\text { Hidro } \\
\text { eléctrica }\end{array}$ & Renovables \\
\hline Canadá & 31.1 & 28.5 & 5.3 & 6.3 & 25.8 & 3.0 \\
\hline Estados Unidos de América & 40.9 & 28.4 & 14.9 & 8.6 & 3.0 & 4.2 \\
\hline $\begin{array}{l}\text { Estados Unidos de América } \\
\text { y Canadá }\end{array}$ & 39.6 & 28.5 & 13.6 & 8.3 & 6.1 & 4.1 \\
\hline Argentina & 36.8 & 48.5 & 1.2 & 1.7 & 11.0 & 0.8 \\
\hline Brasil & 46.1 & 11.2 & 5.6 & 1.2 & 28.4 & 7.5 \\
\hline Chile & 47.9 & 13.5 & 17.5 & 0.0 & 13.2 & 7.9 \\
\hline México & 45.8 & 39.8 & 6.9 & 1.3 & 3.8 & 2.3 \\
\hline América Latina y el Caribe & 45.5 & 21.3 & 4.7 & 0.7 & 23.2 & 4.7 \\
\hline Alemania & 35.8 & 23.1 & 21.3 & 5.1 & 1.3 & 13.4 \\
\hline España & 46.7 & 19.8 & 9.7 & 9.5 & 3.0 & 11.3 \\
\hline Italia & 38.8 & 39.7 & 6.3 & 0.0 & 5.3 & 9.9 \\
\hline Países Bajos & 47.4 & 36.0 & 10.6 & 1.4 & 0.0 & 4.6 \\
\hline Reino Unido & 39.9 & 35.4 & 4.7 & 8.3 & 0.7 & 11.0 \\
\hline Europa & 37.1 & 23.2 & 15.1 & 9.8 & 6.6 & 8.2 \\
\hline $\begin{array}{l}\text { Comunidad de Estados } \\
\text { Independientes }\end{array}$ & 20.8 & 50.5 & 16.1 & 6.7 & 5.8 & 0.1 \\
\hline Oriente Medio & 46.8 & 51.4 & 0.9 & 0.2 & 0.5 & 0.2 \\
\hline África & 43.7 & 27.1 & 20.7 & 0.8 & 6.5 & 1.2 \\
\hline Asia Pacífico & 28.6 & 11.5 & 48.4 & 1.9 & 6.5 & 3.0 \\
\hline
\end{tabular}

Fuente: BP (2018). 
En época de profundos desequilibrios en la economía internacional, la promoción de la EFR se convirtió en una estrategia para impulsar el desarrollo de la industria local y la creación de empleo. Mientras las políticas energéticas se orientan a crear y consolidar los mercados domésticos de EFR (Zhang et al., 2013), las políticas de carácter industrial establecen estrategias para apoyar la competitividad de las empresas locales vinculadas al sector y a su capacidad de crear puestos de trabajo, aumentar las exportaciones y sustituir la importación de bienes y servicios (Lund, 2009; Lewis y Wiser, 2007).

Existen diferencias en la literatura académica acerca de la contribución de la EFR al desarrollo de la industria local. Los estudios realizados en países de la Unión Europea, en general, presentan evidencia de la incidencia positiva de las políticas de promoción de la EFR en el desempeño de la industria doméstica, tanto en materia de exportación, como de creación de empleo (López-Polo et al., 2006; Weiss et al., 2003). Los trabajos destacan el papel del Estado en la creación de mercados domésticos de EFR amplios, sostenibles y estables en el tiempo, que favorecen el fortalecimiento de las empresas locales para su posterior inserción en mercados internacionales, incluso partiendo de una base industrial débil (Kranzl et al., 2006; Lewis y Wiser, 2007; Arentsen et al., 2007; Lund, 2009; Zhang et al., 2013). Además, señalan el papel de las políticas industriales de apoyo directo a la industria doméstica como estrategia efectiva para impulsar su diversificación hacia la EFR y abrir nuevas posibilidades de exportación (Lewis y Wiser, 2007; Lund, 2009; Zhang et al., 2013).

Sin embargo, otros autores señalan que no existe una relación unívoca entre las políticas de promoción de EFR, sus múltiples objetivos -energéticos, económicos y sociales-, y la creación de empleo (Frondel et al., 2010; Pahle et al., 2016), ya que ésta depende del marco regulatorio, la composición de la matriz energética, y la estructura productiva inicial (Lambert y Pereira Silva, 2012).

En el caso de Argentina, el Estado nacional crea políticas de promoción de la EFR desde la década de los setenta, con resultados poco significativos en el mercado energético y la industria local. Tal es así que, en la actualidad, la participación de la EFR en la matriz eléctrica nacional representa menos del $2 \%$ de la potencia instalada $(720 \mathrm{MW}$ en 2017$) .^{2}$ Sin embargo, la modificación del Régimen de Fomento Nacional para el uso de Fuentes Renovables de Energía destinada a la Producción de Energía Eléctrica (Ley 27.191), en

2 A diferencia de otros países, en Argentina la Ley nacional 27.191 no considera EFR a la proveniente de centrales hidroeléctricas con una potencia superior a $50 \mathrm{MW}$, si no a la generada por pequeños aprovechamientos hidroeléctricos (hasta $50 \mathrm{MW}$ ). 
septiembre de 2015, y el cambio de administración del Poder Ejecutivo nacional, en diciembre de ese mismo ańo, marcaron una nueva etapa para la EFR en el país.

Este artículo analiza la política energética y la política industrial en materia de EFR, adoptadas por el gobierno nacional argentino desde finales de 2015 para responder las siguientes interrogantes: 1) ¿qué objetivos tiene la actual política energética en materia de EFR?; 2)¿qué lugar ocupa el desarrollo de la industria nacional en el diseño de esta política?; 3)¿̨ómo se plasma el objetivo de desarrollo de la industria nacional en el diseño y formulación de la política energética y de las políticas industriales que la acompañan?; 4)¿qué resultados son visibles y cómo son evaluados por el sector público y el sector privado?

Para abordar estas preguntas, en la sección 2, se describe cómo ha evolucionado el marco regulatorio de la EFR desde la década de los setenta en Argentina. En la sección 3, se analiza la coherencia de los objetivos y la formulación de las políticas energéticas e industriales de alcance nacional, asociadas a la EFR vigentes desde finales de 2015, para después, en la sección 4, evaluar los aciertos y los desafíos de las políticas a través de los resultados inmediatos que ha tenido el programa RenovAr. Finalmente, se presentan las conclusiones del trabajo atendiendo a las preguntas planteadas.

\section{EVOLUCIÓN DE LAS POLÍTICAS DE PROMOCIÓN DE LA EFR}

Como en el resto de América Latina, el interés por promover la generación de EFR en Argentina data de la década de los setenta, cuando la crisis del petróleo mostró la vulnerabilidad de los países que carecían de recursos energéticos propios. Aunque en esos años Argentina contaba con un adecuado balance energético (Silva Colomer et al., 2014), el gobierno nacional propuso desarrollar una política activa de promoción de la EFR, poniendo especial énfasis en las reservas hidroeléctricas para disminuir la participación relativa del petróleo en la matriz energética (Barrera, 2011). Dicha tendencia se quebró con el golpe de Estado de 1976; no obstante, durante la década de los ochenta, el sector público nacional formuló planes específicos, pero intermitentes, para recolectar información sobre los recursos energéticos renovables disponibles en el país.

En los años noventa, el sector eléctrico adoptó una nueva configuración institucional y empresarial que implicó la privatización de las empresas públi- 
cas del sector y su desintegración vertical en tres actividades: 1) generación, 2) transporte, y 3) distribución. La generación de electricidad fue definida como una actividad económica susceptible de admitir múltiples actores en competencia. Sin embargo, la ausencia de incentivos específicos a la EFR y los bajos precios relativos del gas natural y el petróleo limitaron la instalación de proyectos de generación de EFR en este mercado.

Con la firma del Protocolo de Kioto (1997), Argentina se comprometió a reducir sus emisiones de gases de efecto invernadero, lo que le brindó acceso a nuevas fuentes internacionales de financiamiento para proyectos ambientales sustentables. Lo anterior motivó la sanción, en 1998, de la primera ley específica dirigida a promover la EFR, denominada Régimen Nacional de la Energía Eólica y Solar (Ley 25.019). Dicha ley estableció beneficios impositivos para los proyectos de generación de energía eléctrica de fuentes renovables y un premio o feed in tariffde 10 US\$/MWh. En esta década, las inversiones se materializaron en una capacidad instalada de $28.88 \mathrm{MW}$ de energía eólica, pero la mayoría de estas plantas no entraron formalmente al Sistema Argentino de Interconexión Eléctrica (SADI), sino que destinaron la electricidad generada a la red de distribución local (Recalde et al., 2015).

El débil esquema de beneficios fiscales y el sistema de primas que ofreció la Ley 25.019 desaparecieron con la crisis que atravesó el país en 2001 y la pronunciada devaluación del tipo de cambio. Los costos de operación y mantenimiento de estas plantas, en su mayoría equipadas con tecnología importada, aumentaron y los ingresos de las generadoras comenzaron a deteriorarse debido al creciente atraso de las tarifas eléctricas reguladas por el gobierno nacional (Jacinto et al., 2014).

Para 2006, el Estado nacional formuló un nuevo Régimen de Fomento Nacional para el Uso de Fuentes Renovables de Energía Destinada a la Producción de Energía Eléctrica (Ley 26.190). La ley establecía, por primera vez, una meta de consumo de EFr equivalente al $8 \%$ de la demanda del Mercado Eléctrico Mayorista (MEM) para 2016; otorgó beneficios fiscales y económicos a los proyectos de generación de EFR, adicionales a los considerados en la Ley 25.019, priorizando aquellos proyectos integrados con bienes de capital nacional. Además, creó el Fondo Fiduciario de Energías Renovables, integrado por un recargo adicional a la tarifa de los usuarios del MEM para premiar la generación de energía eléctrica de fuentes renovables.

En octubre de 2009, en el marco de la Ley 26.190, la Secretaría de Energía estableció el programa GENREN que permite la licitación de contratos de abastecimiento de energía eléctrica por $1000 \mathrm{MW}$ de potencia de EFR por un periodo de 15 años y precios nominados en dólares estadounidenses. La se- 
lección de proyectos se realizó utilizando como criterio el precio de oferta, el cronograma de inversión y el porcentaje de componentes de origen nacional en la inversión del proyecto. La primera licitación recibió 51 propuestas de 22 empresas por un total de $1436.5 \mathrm{MW}, 32$ proyectos tenían una potencia de 895 MW y fueron adjudicados en la siguiente manera: $84 \%$ de energía eólica, $12 \%$ de generación térmica con biocombustibles y el resto a Pequeños Aprovechamientos Hidroeléctricos (PAH) y energía Solar Fotovoltaica (SFv).

Esta licitación mostró que existían en el país proyectos con condiciones tecnológicas y ambientales adecuadas para la generación de EFR, así como capacidad técnica y emprendedora para su desarrollo. Sin embargo, las condiciones de entorno de la política energética argentina -bajo nivel de voluntad política, marcos regulatorios débiles, inestabilidad económica y restricciones financieras- condicionaron la efectividad de los instrumentos de política destinados a fomentar la EFR y, en consecuencia, la instalación de los proyectos $\operatorname{adjudicados}^{3}$ (Recalde et al., 2015).

Con el fin de aumentar la generación de electricidad y cumplir el cupo de $8 \%$ de EFR en la matriz eléctrica, en 2011 el gobierno nacional formuló un nuevo mecanismo de contratación de EFr. La Secretaría de Energía de la Nación (Resolución SE 108/2011) reguló la firma de contratos de abastecimiento entre la Compañía Administradora del Mercado Mayorista Eléctrico (CAMMESA) -en representación del MEM- y proyectos de generación de energía eléctrica de fuentes renovables, por un periodo de 15 años y una remuneración en dólares definida en función de los costos fijos y las variables de cada planta. Poco a poco, la matriz de energía eléctrica comenzó a diversificarse con la conexión a la red de nuevos parques eólicos y solares, y en 2015, de dos plantas de biogás.

\section{LA POLÍTICA ENERGÉTICA Y LA POLÍTICA INDUSTRIAL EN MATERIA DE EFR}

La Ley 27.191 estableció las bases para el diseño de un marco regulatorio que impulsara la conformación de un mercado de EFR y fortaleciera el desarrollo de una industria doméstica de tecnologías para la producción EFr. Sin embargo, las normas y los programas formulados a partir de esta ley persiguieron

3 En la actualidad de los proyectos adjudicados en el GENREN se encuentran conectados al MEM: 3 proyectos de energía eólica de $127.4 \mathrm{MW}$ de potencia, 4 de energía solar de $8.2 \mathrm{MW}$, y 2 de biogás de rellenos sanitarios por 16.6 MW de potencia. 
objetivos de política energética y de política industrial difíciles de compatibilizar en el corto plazo y condicionaron la dinámica de inserción de la industria local en las cadenas globales de valor.

A continuación se analizan el entramado de normas y programas diseñados en torno a la Ley 27.191 y los límites de su implementación.

\section{Un nuevo marco regulatorio para la energía eléctrica de fuentes renovables}

El Régimen de Fomento Nacional para el uso de Fuentes Renovables de Energía destinada a la Producción de Energía Eléctrica (Ley 26.190 de 2006 modificada por la Ley 27.191) estableció en 2015 un cronograma de participación de la EFR en el consumo eléctrico y tres instrumentos claves para promover su generación y modificar la configuración del mercado nacional. Los instrumentos incluyen un fondo fiduciario público destinado a brindar garantías y financiamiento a los proyectos, beneficios impositivos, aduaneros y regulatorios, así como un mercado a término de energía eléctrica de fuentes renovables entre privados.

Por un lado, la ley prorrogó hasta 2017 la meta de cubrir 8\% del consumo nacional de energía eléctrica con EFR, según lo establecía la Ley 26.190, y definió un cronograma de consumo obligatorio hasta alcanzar $20 \%$ en 2025 . A diferencia de políticas anteriores y otras propuestas a nivel internacional, el cronograma planteó metas específicas, mensurables y obligatorias que, junto al compromiso del Poder Ejecutivo nacional, otorgan una visión clara sobre la trayectoria de crecimiento de la demanda de efr (IRENA, 2017a). Su cumplimiento demandará la incorporación a la matriz eléctrica de unos $1000 \mathrm{MW}$ de potencia adicional de fuentes renovables anuales, por los próximos 10 años.

No obstante el desafío que representa este cronograma para Argentina, otros países de la región y del mundo se han propuesto metas más exigentes de participación de la EFR en la matriz eléctrica, por ejemplo: 35\% para el 2024 en México (sEner, 2017); 86\% para el 2024 en Brasil (incluyendo grandes hidroeléctricas) (Ministério de Minas e Energia, 2015); y 80\% para el 2050 en Alemania (Arranz, 2016). Estas metas, sin embargo, son el resultado de una trayectoria previa de fuerte impulso a la EFR que en la actualidad se proponen consolidar y profundizar el crecimiento del sector.

Por otra parte, en materia de instrumentos para dinamizar la oferta, la nueva ley amplía los beneficios fiscales, aduaneros y regulatorios que la Ley 26.190 ofrecía a los proyectos de generación de energía eléctrica de fuentes 
renovables, y establece que éstos deben decrecer en el tiempo hasta su extinción en 2025 para acelerar su instalación. Entre los beneficios más relevantes para los fines que busca este trabajo están, por un lado, el hecho que el Estado otorga a los proyectos un certificado fiscal para el pago de impuestos nacionales por un monto equivalente al $20 \%$ del valor del componente nacional de las instalaciones electromecánicas. ${ }^{4}$ Por otra parte, exime a estos proyectos del pago de derechos de importación por los bienes de capital no producidos en el país (hasta el 2022 inclusive, según el Decreto 814/2017). Mientras algunos referentes señalan que este último beneficio retrasa el crecimiento de la industria nacional, otros consideran que la lista de bienes exentos es restrictiva y podría generar cuellos de botella en la etapa de construcción de los primeros proyectos (Siboldi, 2016).

En segundo lugar, la ley propone la creación del Fondo para el Desarrollo de Energías Renovables (FODER) ${ }^{5}$ para ampliar el financiamiento de los proyectos. El fondo fiduciario está destinado a financiar la construcción de proyectos de EFR y otorgar avales y garantías por las obligaciones de pago asumidas por CAMMESA. Los proyectos con mayor porcentaje de componente nacional en la inversión total tienen prioridad en el acceso a este Fondo. Sin embargo, hasta el momento, el FODER no ha actuado como fondo de financiamiento, sino como fondo de garantía, dejando en manos de los privados la búsqueda y negociación del financiamiento.

Para incrementar la confianza de los inversores, el Ministerio de Energía y Minería de la Nación (Minem) firmó acuerdos con el Banco Mundial para que, a opción de los proyectos interesados, respalde el papel del FODER como garante de CAMMESA. Las nuevas políticas públicas se encuentran condicionadas por las políticas previas (Rogge et al., 2017), y esta combinación de garantías es una estrategia efectiva para disminuir la incertidumbre asociada a la capacidad de pago de CAMMESA y al funcionamiento del sector eléctrico.

Por último, la ley define el marco para la creación de un mercado a término de energía eléctrica de fuentes renovables entre privados al establecer que los grandes usuarios del MEM (con demandas de potencia de $300 \mathrm{Kw}$ o más)

4 Este certificado fiscal es expedido cuando la integración de componentes de origen nacional es mayor al $60 \%$ o si el porcentaje es menor, cuando se demuestra efectivamente la inexistencia de producción nacional, la que en ningún caso podrá ser inferior a $30 \%$.

5 El Fondo está integrado por cargos específicos a la demanda de energía eléctrica, recursos provenientes del Tesoro Nacional -equivalentes a no menos del 50\% del ahorro efectivo en combustibles fósiles por la incorporación de generación de EFR en el año previo-, y otros recursos nacionales e internacionales. 
deben alcanzar efectiva e individual las metas de consumo de EFR definidas por la ley. Los casi 7500 usuarios alcanzados por esta medida -el $90 \%$ con un consumo menor a $1 \mathrm{MW}$, según la Asociación de Grandes Usuarios de Energía Eléctrica de la República Argentina- podrán realizar contrataciones individuales de energía eléctrica, autogenerar o cogenerar, o participar en el mecanismo de compras conjuntas que realiza CAMMESA. Esta última opción, sin embargo, incluirá cargos en concepto de comercialización y administración sujetos a la demanda de cada usuario y el cupo de EFR vigente.

Según referentes del sector privado, en el corto plazo sólo las empresas con una demanda de potencia mayor a $10 \mathrm{MW}$ (alrededor de 53) optarán por no participar de las compras conjuntas a CAMMESA, pero con el tiempo, a medida que los cargos se encarezcan, la mayoría de las empresas optará por contratos en el mercado a término. Este mercado representa una oportunidad para los generadores de EFR, ya que aun cuando no ofrece las mismas garantías y avales que la licitación pública, permite atomizar el riesgo en distintos clientes, muchos de alcance internacional, y plantear un sendero de crecimiento escalonado, en la medida que la demanda se expanda. Para los grandes usuarios representa una posibilidad de reducir sus costos -sobre todo con la energía eólica y SFV- y asegurar su abastecimiento.

\section{Los objetivos de política y su formulación}

En 2016, el MINEM estableció como objetivos centrales de su gestión en materia energética mejorar la eficiencia de la oferta y de la demanda, aumentar la seguridad del sistema, y desarrollar un mercado más competitivo. Para alcanzar estas metas, el organismo planteó tres líneas centrales de trabajo: 1) incrementar la producción nacional de gas natural y petróleo -en retroceso durante los años previos (Ceppi, 2018)-; 2) mejorar la eficiencia en el consumo, y 3) ampliar la capacidad de producción y transporte de energía eléctrica (MINEM, 2016). El apoyo a la generación de EFR -con costos de operación estables y relativamente independientes de los mercados internacionales- se incluyó como parte de estos lineamientos, debido a que su incorporación en el sistema eléctrico nacional contribuye a cumplir varios de los objetivos propuestos como ampliar la potencia disponible, sustituir la importación de energía eléctrica y combustibles fósiles, y disminuir la vulnerabilidad externa del sistema.

En línea con estos objetivos, la Subsecretaría de Energías Renovables, creada en 2016, es la encargada de definir la velocidad, dirección y mecanismos 
de inserción de la EFR en el sistema eléctrico, considerando tanto su diversificación tecnológica como su distribución geográfica (Subsecretaría de Energías Renovables, 2016). Los objetivos del MINEM y la Subsecretaría responden a las metas planteadas por la Ley 27.191 -competitividad del sistema energético, desarrollo de mercados asociados a la EFR, diversidad tecnológica y distribución geográfica-, pero la ley también menciona la necesidad de desarrollar industrias asociadas a estas tecnologías. El desarrollo de proveedores domésticos, sin embargo, no forma parte de los objetivos centrales de la Subsecretaría. Aun así, diversos actores públicos de esta y otras áreas del MINEM destacaron públicamente el papel de los proyectos de EFR como mecanismo para canalizar nuevas inversiones extranjeras directas en el país e impulsar la producción local y el empleo.

Una de las principales estrategias desplegada por el MINEM, para ampliar la potencia y participación de la EFR en la matriz eléctrica, es la implementación del programa RenovAr, que brinda el marco para la compra, mediante convocatoria pública, de energía eléctrica de fuentes renovables. En mayo de 2016, el gobierno nacional lanzó la ronda 1 de este programa y en octubre de 2017 la ronda 2. En la primera ronda licitó contratos de abastecimiento de energía eléctrica por $1000 \mathrm{MW}$ de potencia de EFR, estableciendo cupos para cada tipo de tecnología. En la segunda ronda licitó contratos por $1200 \mathrm{MW}$ de potencia con cupos por tecnología y por región para la energía eólica y solar. En la primera convocatoria se presentaron 123 proyectos que ofrecieron $6346 \mathrm{MW}$, de los cuales 105 (5 209 MW) pasaron la evaluación formal, técnica, económica y legal, y 59 fueron adjudicados (2 434.7 MW). En la siguiente ronda se presentaron 228 proyectos con una potencia total de 9403 MW, calificaron 194 y se adjudicaron 88 (1 942.3 MW).

Los proyectos fueron seleccionados en función del Precio de Oferta Ajustado, ${ }^{6}$ el cual debía ser menor al Precio Máximo de Adjudicación definido por el MiNem para cada tecnología. Sólo en caso de empate técnico entre dos proyectos se priorizó el de mayor puntaje de Componente Nacional Declarado (CND). ${ }^{7}$ En la práctica, este criterio otorgó un papel marginal al CND en el proceso de selección de los proyectos, a diferencia de lo que ocurre en licitaciones públicas de otros países, donde los objetivos socioeconómicos de la política energética en materia de EFR se reflejan directo en su diseño. En

6 Se calcula a partir del precio ofertado para el proyecto, el factor de pérdidas asociado al punto de interconexión y el plazo programado de habilitación comercial.

7 El CND refleja la participación de componentes de origen nacional en las instalaciones electromecánicas de los proyectos, excluyendo la obra civil, según lo define la Ley 27.191. 
Sudáfrica, por ejemplo, el programa de licitación evalúa los proyectos otorgando una ponderación de $70 \%$ al precio y de $30 \%$ al cumplimiento de objetivos socioeconómicos como la creación de empleo directo, el componente nacional, y la estructura de propiedad (Pahle et al., 2016).

La transición hacia un modelo económico ambiental y social sostenible, basado en las capacidades tecnológicas, así como en la innovación local, demanda una política industrial activa centrada en abordar los problemas de coordinación que limitan la diversificación y la difusión de la tecnología (Bárcena, 2017). En un inicio, en Argentina la política industrial hacia el sector de la EFr se apoyó en el Programa de Desarrollo de Proveedores (PRODEPRo) del Ministerio de Producción (MP). Este programa selectivo ofrece financiamiento a tasa subsidiada, aportes no reintegrables, y asistencia técnica a industrias consideradas estratégicas, como la asociada a la generación de EFR. De esta forma, busca facilitar la adquisición de capital físico, el desarrollo, la certificación y la homologación de productos, las reformas productivas, y la capacitación de los recursos humanos.

Las actividades intensivas en el uso de recursos naturales -como la producción de EFR- demandan, con mayor o menor intensidad, innovaciones adaptativas y procesos de búsqueda continua de soluciones para aumentar su productividad -por lo general, limitada por restricciones físicas- y dar respuesta al crecimiento de la demanda (Pérez et al., 2014). En esta línea, el Acuerdo por la Producción y el Empleo: Principales lineamientos para el sector de Energías Renovables, firmado en octubre de 2017 por representantes del sector público ${ }^{8}$ y el sector privado -sindicatos, asociaciones y cámaras empresariales-, ${ }^{9}$ busca incentivar la innovación y la competitividad de las empresas domésticas del sector. ${ }^{10}$ El documento plantea una visión de mediano plazo (2018-2023) y un conjunto de acciones de política de tipo funcional,

MINEM, MP, Ministerio de Trabajo, Empleo y Seguridad Social, Administración Federal de Ingresos Públicos, Agencia Argentina de Inversiones y Comercio Internacional, y Dirección General de Aduanas.

9 Asociación de Industriales Metalúrgicos de la República Argentina, Cámara de Industriales de Proyectos e Ingeniería de Bienes de Capital, Cámara Eólica Argentina, Unión Obrera Metalúrgica, Sindicato de Luz y Fuerza, Unión Obrera de la Construcción de la República Argentina.

El Estado estima que hay 4 mil trabajadores empleados en actividades conexas a la EFR, más de 110 empresas proveedoras de la cadena eólica y solar fotovoltaica, 5 fabricantes de calderas para biomasa y alrededor de 50 proveedores locales de equipamiento eléctrico de uso transversal a todas las tecnologías asociadas a la EFR. 
horizontal y vertical orientadas al desarrollo y la transferencia de tecnología (Lall y Teubal, 1998).

Por un lado, en línea con los objetivos del MINEM, el acuerdo propone aumentar la generación de EFR priorizando la competitividad en precios de los proyectos. Al mismo tiempo, busca aumentar la integración de componentes locales en los proyectos y la transferencia de tecnología hacia las empresas domésticas, con la finalidad de impulsar su inserción en cadenas globales de valor. Esto representa un desafío para la política industrial que ha visto limitados sus espacios de acción por la mayor apertura de la economía a la competencia internacional.

Por otro lado, las acciones que plantea el acuerdo (véase cuadro 2) se orientan a impulsar la creación de un mercado estable de energía eléctrica de fuentes renovables y el crecimiento de dos sectores considerados claves por sus capacidades y su proximidad con las tecnologías de generación de EFR: la industria metalmecánica y los proveedores de equipos y componentes eléctricos. En particular, el acuerdo se propone fortalecer las cadenas de fabricación de aerogeneradores y paneles solares y alcanzar una participación del 50 y el 30\%, respectivamente, de componentes nacionales para 2023.

La política industrial debe promover la producción de bienes y servicios, así como la adopción de procesos que generen nuevas capacidades y conocimiento experimental y tácito. Muchas de estas capacidades se plasman en rutinas de ingeniería, de diseño y de gestión que se desarrollan y reproducen por medio de la experiencia, y que trabajadores, técnicos y empresarios ejecutan de manera más o menos automática. Producto de esta inercia, el aprendizaje y la construcción de nuevas capacidades son un proceso incremental en relación con el espacio tecnológico en que se mueven las empresas y dependiente de su trayectoria tecnológica previa (Porcile y Martins, 2017). Estas condiciones son necesarias para que las empresas generen diseños y marcas propias en alta tecnología (Lee, 2013). De lo contrario, el proceso de innovación se realiza a través de la importación de bienes de capital, la obtención de licencias o patentes y/o la especialización en segmentos de ensamble con poco contenido tecnológico en cadenas globales de producción (Yoguel et al., 2017). 
Cuadro 2. Caracterización de las acciones de política industrial del Acuerdo (2017)

\begin{tabular}{|c|c|c|}
\hline Programa/Etapa & Instrumento & Objetivo \\
\hline RenovAr & $\begin{array}{l}\text { Licitación pública de contratos de } \\
\text { abastecimiento de energía eléctrica de } \\
\text { fuentes renovables. }\end{array}$ & $\begin{array}{l}\text { Desarrollo de mercado de energía eléctrica } \\
\text { de fuentes renovables. }\end{array}$ \\
\hline Ley 27.191 & Incentivos fiscales a proyectos de EFR. & $\begin{array}{l}\text { Desarrollo de mercado de energía eléctrica } \\
\text { de fuentes renovables. }\end{array}$ \\
\hline Ley 27.191 & $\begin{array}{l}\text { Certificado fiscal por integración de } \\
\text { componente nacional. }\end{array}$ & Desarrollo de proveedores locales. \\
\hline Ley 27.191 & Esquema arancelario de importación. & $\begin{array}{l}\text { Alcanzar precios competitivos en toda la } \\
\text { cadena de suministro. }\end{array}$ \\
\hline ReProER & $\begin{array}{l}\text { Registro de fabricantes y proveedores de } \\
\text { proyectos de EFR. }\end{array}$ & Desarrollo de proveedores locales. \\
\hline En proceso & $\begin{array}{l}\text { Financiamiento a proyectos de generación } \\
\text { de EFR. }\end{array}$ & Participación nacional en proyectos de EFR. \\
\hline \multirow[t]{2}{*}{ PRODEPRO } & Financiamiento a proveedores. & $\begin{array}{l}\text { Promover la integración de proveedores } \\
\text { locales en cadenas globales de valor. }\end{array}$ \\
\hline & Asistencia técnica a proveedores & \\
\hline \multirow[t]{2}{*}{ En proceso } & $\begin{array}{l}\text { Fortalecimiento del "Sistema Nacional de } \\
\text { Innovación". }\end{array}$ & $\begin{array}{l}\text { Impulsar la transferencia y desarrollo de } \\
\text { nuevas tecnologías. }\end{array}$ \\
\hline & $\begin{array}{l}\text { Acuerdos de cooperación con tecnólogos } \\
\text { extranjeros. }\end{array}$ & \\
\hline
\end{tabular}

Fuente: elaboración propia con base en el Ministerio de Producción (2017).

\section{AlgunOS RESUlTAdos de la POLÍtiCa ENERGÉTICA Y LA POLÍTICA INDUSTRIAL}

Aunque los proyectos adjudicados en el programa RenovAr se encuentran en la etapa de construcción (ronda 1) o en la etapa previa a la firma de los contratos (ronda 2), su análisis permite comprender la orientación y el alcance de la política energética del MINEM, así como también los desafíos que enfrenta la política industrial para potenciar el desarrollo de estas tecnologías en el país. A continuación, se analizarán los resultados del programa en materia de diversificación tecnológica, el precio de la energía eléctrica y la participación de los componentes de origen nacional en las instalaciones electromecánicas de los proyectos. 


\section{Diversificación tecnológica}

La transición energética desde una matriz eléctrica altamente dependiente del gas natural hacia una matriz diversificada y sustentable requiere una política que guíe este proceso, asegurando un balance continuo entre los recursos renovables disponibles y la sostenibilidad del sistema. La composición tecnológica de la matriz eléctrica -que condiciona la relación entre energía de base y energía intermitente-, ${ }^{11}$ la ubicación de los proyectos en relación con la disponibilidad de los recursos renovables y de los nodos de transporte, y la incidencia de ambos en la eficiencia de los proyectos y en el precio de la energía eléctrica condicionan la velocidad y dirección de la transición energética.

Respondiendo a estos condicionantes, el programa RenovAr estableció cupos de adjudicación para cada tipo de EFR, lo que se reflejó en una creciente diversificación tecnológica de la potencia adjudicada en relación con licitaciones previas. Aun así, la potencia adjudicada por el RenovAr corresponde en sus mayoría a energía eólica (54\%) y SFV (39.8\%), mientras que la participación de los PAH $(0.7 \%)$ y las plantas de bioenergía -biomasa y biogás- $(5.4 \%)$ se encuentran rezagada (véase cuadro 3 ).

Cuadro 3. Potencia de proyectos de generación de EFR adjudicados en licitaciones según tecnología (en MW)

\begin{tabular}{lrcc}
\hline \multicolumn{1}{c}{ Tecnología } & Genren & Renovar 1 & Renovar 2 \\
\hline Eólica & 754.0 & 1472.9 & 893.4 \\
Solar fotovoltaica & 20.0 & 927.2 & 816.3 \\
PAH $<30 / 50 \mathrm{MW}$ & 11.0 & 11.4 & 20.1 \\
Térmica biocombustible & 110.4 & $\mathrm{NL}$ & $\mathrm{NL}$ \\
Biogás & $\mathrm{NA}$ & 8.6 & 69.3 \\
Biomasa & $\mathrm{NA}$ & 14.5 & 143.2 \\
\hline Total & 895.4 & 2434.7 & 1942.3 \\
\hline
\end{tabular}

Notas: NA Tecnología no adjudicada; NL Tecnología no licitada.

Fuente: elaboración propia con base en los datos de licitación del MINEM (2017).

La energía de base es producida de manera continua, con costos económicos aceptables para el mercado, por lo que cubre la "base" de la curva de demanda. Las oscilaciones de la demanda por encima de esta "base" son cubiertas por la energía producida por tecnologías con mayor nivel de intermitencia, según su disponibilidad y sus costes. La intermitencia de esta energía se debe a que ciertas centrales de generación de electricidad sólo pueden funcionar cuando el recurso -el sol, el viento, las mareas- está disponible. 
Los PAH son una fuente de energía difundida en el país -en 2011 ya había $380 \mathrm{MW}$ instalados y hoy hay $488 \mathrm{MW}-$, promovida en décadas anteriores junto a grandes centrales hidroeléctricas. Aunque existe un amplio margen para ampliar la potencia de PAH a lo largo del país, estos proyectos por lo general se encuentran en zonas aisladas lo que incrementa su costo por MWh entregado al MEM. La bioenergía, por otro lado, es una tecnología madura a nivel internacional, pero incipiente en el país; con un gran potencial por la disponibilidad de recursos agropecuarios y agroindustriales, pero con una cadena de valor compleja en su organización y costos. Además, ambas tecnologías se caracterizan por potencias medias relativamente bajas en relación con la energía eólica y solar.

El número de proyectos adjudicados también aumentó entre las rondas 1 y 2 del RenovAr -pasó de 59 a 88- y, en especial, creció la participación de proyectos de biomasa (de 2 a 16) y de biogás (de 6 a 34). La disminución de la potencia mínima requerida para estas tecnologías (de $1 \mathrm{MW}$ en la ronda 1 a $0.5 \mathrm{MW}$ en la ronda 2) y la aplicación de incentivos de precio a los proyectos de menor escala en la ronda 2 impulsó un aumento en el número de proyectos con niveles de potencia bajos, los que permiten hacer un uso más eficiente de la red de transporte instalada y un mayor uso de biomasa residual.

\section{Precios}

En la ronda 1 de la licitación del RenovAr, los precios ofertados resultaron más bajos que los Precios Máximos Adjudicados -desconocido hasta el momento de apertura de los sobres- y menores que las expectativas del sector privado y de los grandes usuarios - con obligaciones de consumo de EFR por la Ley 27.191- que tenían como referencia los precios de la licitación del GENREN (véase cuadro 4). Además, $78 \%$ de los proyectos fueron adjudicados con precios que no superaron el costo monómico medio ${ }^{12}$ de generación del sistema eléctrico nacional en ese año (71.3 US\$/MWh en 2016). ${ }^{13}$

El Costo Medio Monómico es la suma de los costos representativos de producción (fijos y variables) de energía eléctrica en el MEM, divida la demanda abastecida total, en un periodo de control.

13 Estos precios no consideran los ajustes por el índice del Factor de Incentivo que premia la habilitación temprana de los proyectos y decrece con el tiempo y, por otro lado, el Factor de Ajuste anual que incrementa el precio entre 1.5 y 2 puntos anuales. En total, se estima que el precio promedio es alrededor de $20 \%$ más alto, lo que implicaría que $49 \%$ de los proyectos no superen el costo monómico mencionado para esa fecha. 
Cuadro 4. Precio medio de proyectos adjudicados en licitaciones según tecnología. En US\$ $/ M W^{\star}{ }^{\star}$

\begin{tabular}{lrrr}
\hline \multicolumn{1}{c}{ Tecnología } & Genren & Renovar 7 & Renovar 2 \\
\hline Eólica & 127.0 & 56.2 & 40.9 \\
SFV & 570.0 & 57.0 & 42.8 \\
PAH <30/50 MW & 162.0 & 105.0 & 98.9 \\
Térmica biocombustible & 287.0 & $\mathrm{NL}$ & $\mathrm{NL}$ \\
Biogás & $\mathrm{NA}$ & 154.0 & 154.7 \\
Biomasa & $\mathrm{NA}$ & 110.0 & 117.2 \\
\hline
\end{tabular}

Notas: *Los precios corresponden al promedio ponderado de los Precios de Oferta Ajustados; NA Tecnología no adjudicada; NL Tecnología no licitada.

Fuente: elaboración propia con base en los datos de licitación del MINEM (2017).

Estos resultados se enmarcan, por una parte, en una tendencia a la baja de los precios de la energía eólica y solar a nivel internacional, impulsada por la disminución del costo de estas tecnologías. ${ }^{14}$ En Argentina, por otra parte, los cambios en el entorno político y económico, la competencia creada por el sistema de licitaciones y las condiciones de contratación y garantías que brindó el RenovAr disminuyeron el costo de financiamiento y ampliaron su disponibilidad a nivel internacional. La menor incertidumbre económica y financiera se reflejó en una caída en el porcentaje de proyectos adjudicados que solicitaron la garantía del Banco Mundial del 54\% en la ronda 1 a $31.8 \%$ en la ronda 2. Estas condiciones permitieron que los Precios de Oferta Ajustados alcanzaran niveles comparables a los de mercados con más trayectoria (véase gráfica 1).

Además, al priorizarse el precio de los proyectos, primó como criterio de selección su eficiencia, esto es la articulación entre la tecnología y la calidad del recurso renovable disponible, dejando en un segundo plano factores como la participación de componentes de industria nacional, la distribución geográfica -parcialmente en la segunda ronda-, o la cercanía a los centros de consumo.

14 Según IRENa (2017c), el costo promedio normalizado de la electricidad a nivel mundial disminuyó $62 \%$ para la energía SFv y $21 \%$ para la solar entre 2010 y 2016. 
Gráfica 1. Precios adjudicados en licitaciones según tecnología y país. En US\$/MWh (2016)

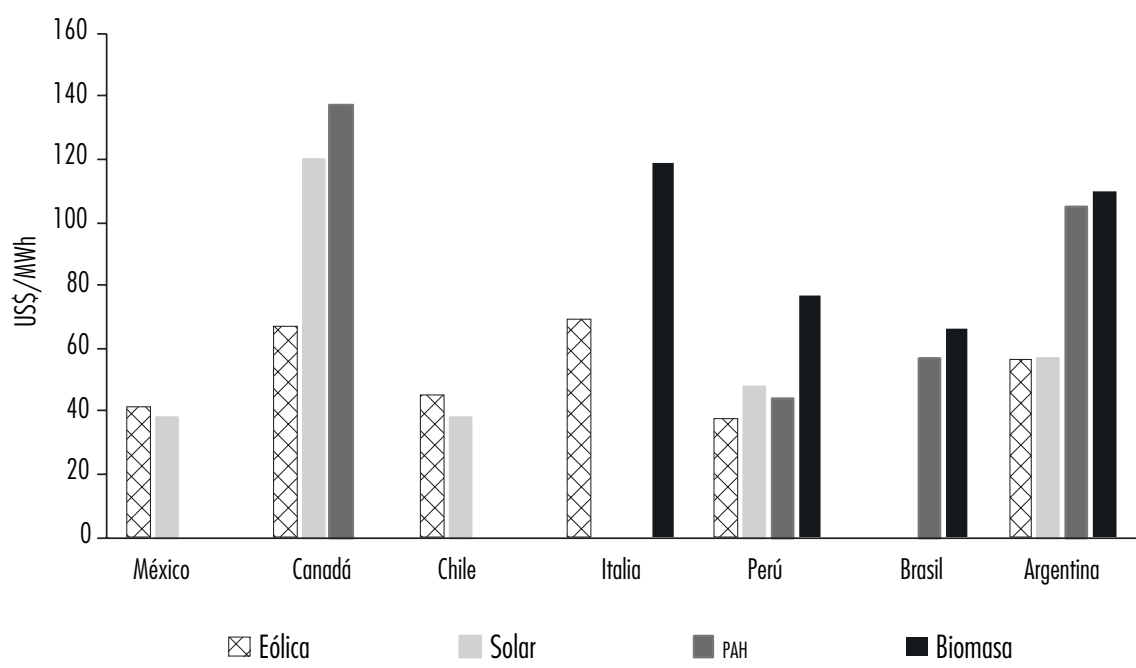

Fuente: elaboración propia en base a datos de IRENA (2017b).

El nivel de precio de la energía eólica y solar en el país, aunque positivo para la competitividad del sistema energético, representa un desafío para el crecimiento del mercado. Según referentes del sector privado, si la tendencia a la baja se profundiza sólo los proyectos de mayor escala, con más precisión en los costos, y más desarrollados -con menores riesgos de contingencias y más conocimiento de las características del recurso renovable disponible- lograrán ser adjudicatarios en nuevas licitaciones.

\section{Componente Nacional Declarado}

La inversión en proyectos de EFR ofrece un amplio margen para promover la creación de empleo a través de la participación de la industria local en las etapas de desarrollo, instalación, y operación y mantenimiento de estos proyectos. La etapa de desarrollo tecnológico se caracteriza por crear empleo estable y altamente especializado; así como la etapa de instalación demanda de manera intensiva mano de obra local, temporaria y también especializada; y la etapa de operación y mantenimiento requiere empleo local, estable y medianamente especializado (oIT, 2011; Lambert y Pereira Silva, 2012). 
No obstante, el impacto real en el empleo de los proyectos de generación de EFR depende de la inserción de la industria local en las primeras etapas de la cadena, ya que las últimas etapas son más intensivas en servicio, pero poseen menor peso en el valor agregado global (Sica, 2016).

La integración de bienes de origen nacional de los proyectos adjudicados del programa RenovAr -computada por los proyectos como CND para la obtención de beneficios fiscales- mostró una amplia dispersión en las rondas 1 y 2 y una tendencia dispar entre ambas (véase gráfica 2). De hecho, la integración de CND por tecnología no se encuentra correlacionada con el precio ofertado y tampoco muestra una relación estable entre las rondas con la escala de los proyectos.

Sólo los proyectos eólicos aumentaron el porcentaje promedio de CND (de 11 a 35\%) y su dispersión. Los proyectos con una integración cercana a 100\% de CND son escasos y corresponden en su mayoría a proyectos que proponen ampliar su capacidad instalada.

Gráfica 2. Porcentaje de CND en licitaciones según tecnología (2016-2017)

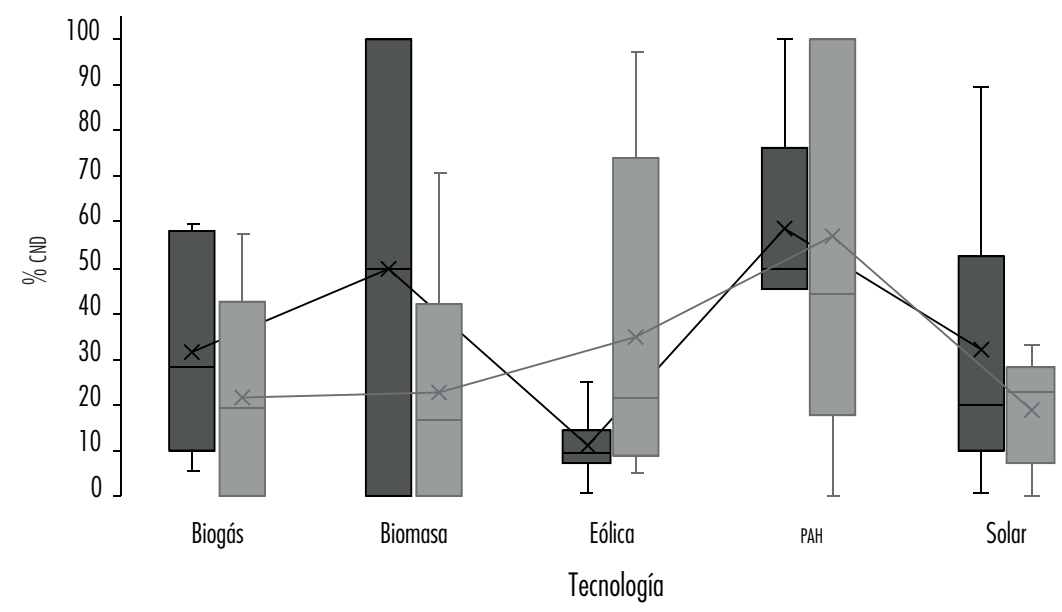

$\square$ Ronda $1 \square$ Ronda 2

Fuente: elaboración propia en base a datos del MINEM (2017). 
La tendencia decreciente de la media de CND, aunque de corto plazo, cuestiona la idea de que es posible aumentar la integración de componentes nacionales en los proyectos de EFR sin aplicar criterios en el proceso de licitación que ponderen su incorporación y/o políticas de asistencia o financiamiento que "premien" a quienes eligen involucrarse en el desarrollo de cadenas integrales de valor o en procesos de transferencia tecnológica. Diversos referentes de empresas dedicadas al diseño e instalación de proyectos en el país manifiestan que, en general, no existen proveedores locales que ofrezcan productos específicos para estas tecnologías.

El gobierno de Brasil, por ejemplo, implementó políticas que promueven el contenido local en los proyectos de energía eólica como el Programa de Incentivo a las Fuentes Alternativas de Energía Eléctrica (PROINFA) que brinda financiación del Banco Nacional de Desarrollo Económico y Social si al menos 60\% del parque eólico contiene piezas locales (Freier, 2016). Estas experiencias muestran que es posible aplicar metas de componentes nacionales de manera gradual y escalonada en mercados de energía con potencial suficiente (Lewis y Wiser, 2007). En las licitaciones realizadas por este país la energía eólica posee una participación significativa y también sus industrias asociadas (Puerto Rico y Sauer, 2015).

Actores públicos y referentes del sector privado coinciden en promover el surgimiento y desarrollo de una industria nacional vinculada al mercado de la EFR. Sin embargo, descartan la posibilidad de imponer una participación mínima de componentes de origen nacional en los proyectos en la etapa de conformación del mercado, bajo el argumento de que aumentarían los Precios de Oferta Ajustados y que el crecimiento del mercado será el impulsor de la dinámica de innovación de la industria nacional. Otros referentes del sector privado, sin embargo, cuestionan las posibilidades de penetración de la industria doméstica en el sector de la EFR en las condiciones actuales. La existencia de grandes jugadores internacionales en el terreno de las tecnologías eólicas y SFV, por un lado, incrementa la competencia y, por otro, impone altos estándares de calidad en la producción de los bienes locales que podrían integrarse en la cadena de valor. En otras palabras, el contexto actual exige niveles de competitividad que la industria local aún no ha desarrollado en el sector de la EFR y que sólo podrá alcanzar si logra disminuir la distancia cognitiva y tecnológica entre los jugadores internacionales y las empresas locales. 


\section{CONCLUSIONES}

Este estudio se propuso analizar el marco regulatorio de la EFR vigente en Argentina, atendiendo a sus objetivos, su formulación e implementación, y el alcance de sus resultados. Así también, analizó el papel que las políticas orientadas a la EFR otorgan a la promoción de la industria nacional y su articulación con las políticas industriales que las acompañan.

Es así como se permite afirmar que las políticas públicas orientadas a promover la EFR en el país se encuentran alineadas con los objetivos de la política energética nacional, en especial con la mejora de la seguridad energética y la competitividad del sistema eléctrico en el mediano plazo. La Ley 27.191 brinda las condiciones normativas para promover la ampliación de la potencia eléctrica instalada mediante plantas de generación de EFR con costos de operación, en muchos casos, menores que los costos de otras tecnologías de generación de energía eléctrica. Las normas reglamentarias financieras, impositivas, aduaneras y regulatorias formuladas por el gobierno nacional a partir de esta ley convocaron a un gran número de proyectos de generación de energía eléctrica de fuentes renovables a la licitación pública, con precios de oferta por debajo de los precios máximos establecidos por el RenovAr y de los esperados por el sector privado.

El precio de oferta de la energía eléctrica ha sido el principal criterio de selección de estos proyectos, lo cual coincide con la disminución del precio medio de la EFR ofertada - propiciando la competitividad del sistema eléctrico nacional-, pero condiciona de forma negativa la participación de la industria nacional en la integración de los proyectos. La decisión del MINEM de utilizar el FODER para disminuir la incertidumbre de los inversores, y no para financiar aquellos proyectos con mayor porcentaje de componente nacional, refuerza la orientación de la política energética en detrimento de los objetivos de la política industrial.

La posición de la industria nacional en los objetivos del MINEM contrasta con el lugar que informalmente funcionarios y políticos otorgan a los proyectos de EFR como mecanismo para impulsar la producción local y el empleo. Este proceso parece descansar sobre todo en las políticas sectoriales que el MP fórmula para promover la transferencia de capacidades, la difusión de tecnología y la innovación en el terreno de la EFR, con especial foco en la industria metalmecánica y eléctrica, y en las cadenas de valor eólica y sFv. Los resultados inmediatos muestran un panorama complejo para los proveedores domésticos, con una participación de componentes nacionales relativamente baja y 
sumamente variable en los proyectos de EFr. Su efectividad en el mediano plazo dependerá del alcance de estas políticas y de las capacidades de la industria para generar conocimiento experimental y tácito que impulse procesos de innovación a nivel nacional.

\section{BIBLIOGRAFÍA}

Arentsen, M., Bechberger, M., Di Nucci, M., Midttun, A., Casale, C. y Klemenc, A. (2007), "Renewable Energy and Liberalisation in Selected Electricity Markets-Forum Final Report”, CSTM Studies and Reports, núm. 318.

Arranz, I. (2016), La transición energética en Alemania. Energiewende, Berlín, ICEX.

Bárcena, A. (2017), "Prólogo", en M. Cimoli (ed.), Políticas industriales y tecnológicas en América Latina, Santiago, CEPAL-BUz/GIz.

Barrera, M. (2011), "La diversificación de la matriz energética", Voces en el Fénix: Ultimátum a la tierra, vol. 2, núm. 10, Facultad de Ciencias Económicas, Universidad de Buenos Aires.

Brian Arthur, W. (1989), "Competing Technologies, Increasing Returns, and Lock-in by Historical Events”, The Economic Journal, vol. 99, núm. 394, Royal Economic Society, May.

вр (2018), Base de datos Statistical Review of World Energy, June. Disponible en $<$ http://www.bp.com/statistical review $>$

Cecere, G., Corrocher, N., Gossart, C. y Ozman, M. (2014), "Lock-in and Path Dependence: an Evolutionary Approach to Eco-innovations", Journal of Evolutionary Economics, 24. DOI <https://doi.org/10.1007/s00191014-0381-5>

Ceppi, N. (2018), "Política energética argentina: un balance del periodo 2003-2015", Problemas del Desarrollo. Revista Latinoamericana de Economia, vol. 49, núm. 192, México, IIEc-unam. Disponible en <https://www. probdes.iiec.unam.mx/numeroenpdf/192_v49/02artCeppi.pdf>

Decreto 814/2017, Derecho de Importación Extrazona. Alícuotas. Boletín Oficial de la República Argentina, Buenos Aires (10 de octubre de 2017).

Frantzeskaki, N. y Loorbach, D. (2010), "Towards governing Infrasystem Transitions: reinforcing Lock-in or facilitating Change?”, Technological Forecasting and Social Change, 77(8). DOI <https://doi.org/10.1016/j.techfore.2010.05.004>

Freier, A. (2016), "La situación de la cooperación energética entre Argentina y Brasil en el área de la energía renovable: ¿Integración, difusión o frag- 
mentación?", Relaciones Internacionales, núm. 51. Disponible en <https:// revistas.unlp.edu.ar/RRII-IRI/article/view/2951/2678>

Frondel, M., Ritter, N., Schmidt, C. y Vance, C. (2010), "Economic Impacts from the Promotion of Renewable Energy Technologies: The German Experience", Energy Policy, 38(8). DOI <https://doi.org/10.1016/j. enpol.2010.03.029>

IRENA (2017a), Rethinking Energy 2017: Accelerating the Global Energy Transformation, International Renewable Energy Agency, Abu Dhabi.

(2017b), Featured Dashbord - Policy. IRENA. Disponible en $<$ resourceirena.irena.org/gateway/dashboard/?topic $=1021$ ysubTopic $=1062>$

(2017c), Featured Dashbor-Costs. IRENA. Disponible en $<$ http://resourceirena.irena.org/gateway/dashboard/?topic=3ysubTopic=1057>

Jacinto, G., Clementi, L., Carrizo, S. y Nogar, L. (2014), "Vientos para el cambio. Territorios, energía eólica y cooperativas de electricidad en el sur bonaerense", Revista Transporte y Territorio, núm. 11. Disponible en <http://revistascientificas.filo.uba.ar/index.php/rtt/article/view/656/635>

Jacobs, D., Marzolf, N., Paredes, J.R., Rickerson, W., Flynn, H., BeckerBirck, C. y Solano-Peralta, M. (2013), "Analysis of Renewable Energy Incentives in the Latin America and Caribbean Region: The Feed-in Tariff Case", Energy Policy, 60(C). DOI <https://doi.org/10.1016/j.enpol. 2012.09.024>

Kranzl, L., Stadler, M., Huber, C., Haas, R., Ragwitz, M., Brakhage, A., Gula, A. y Figorski, A. (2006), "Deriving Efficient Policy Portfolios promoting Sustainable Energy Systems: Case Studies applying Invert Simulation Tool", Renewable Energy, 31. DoI <https://doi.org/10.1016/j. renene.2005.11.005>

Lall, S. y Teubal, M. (1998), "Market-Stimulating Technology Policies in Developing Countries: A Framework with Examples from East Asia”, World Development, vol. 26, núm. 8.

Lambert, R.J. y Pereira Silva, P. (2012), "The Challenges of determining the Employment Effects of Renewable Energy", Renewable and Sustainable Energy Reviews, 16. DOI <https://doi.org/10.1016/j.rser.2012.03.072>

Lee, K. (2013), Schumpeterian Analysis of Economic Catch-up: Knowledge, Path-creation, and the Middle-income Trap, Cambridge, MA, Cambridge University Press.

Lewis, J. y Wiser, R. (2007), "Fostering a Renewable Energy Technology Industry: An International Comparison of Wind Industry Policy Support Mechanisms", Energy Policy, 35. DOI <https://doi.org/10.1016/j. enpol.2006.06.005> 
Ley N ${ }^{\circ} 25.019$, Declárase de interés nacional la generación de energía eléctrica de origen eólico y solar en todo el territorio nacional. Boletín Oficial de la República Argentina, Buenos Aires (26 de octubre de 1998).

Ley N ${ }^{\circ}$ 27.191. Modificaciones a la Ley 26190. Régimen de Fomento Nacional para el uso de Fuentes Renovables de Energía destinada a la Producción de Energía Eléctrica. Modificación. Boletín Oficial de la República Argentina, Buenos Aires (21 de octubre de 2015).

Ley $\mathrm{N}^{\circ}$ 26.190, Régimen de Fomento Nacional para el uso de fuentes renovables de energía destinada a la producción de energía eléctrica. Boletín Oficial de la República Argentina, Buenos Aires (2 de enero de 2007).

López-Polo, A., Suna, D. y Haas, R. (2006), "An International Comparison of Market Drivers for Grid connected PV Systems", Proc. 21st European Photovoltaic Solar Energy Conference, Dresden, Germany.

Lund, P.D. (2009), "Effects of Energy Policies on Industry Expansion in Renewable Energy", Renewable Energy, 34(1). DOI <https://doi.org/10.1016/j. renene.2008.03.018>

Ministerio de Energía y Minería (MINEM) (2016), Argentina-Energy for Growth. For the Institute of the Americas, La Jolla, May 26. Recuperado de <www.energia.gob.ar/contenidos/archivos/Reorganizacion/planeamiento/publicaciones/IOA-La\%20Jolla-May2016.pdf>

(2017), Proyectos adjudicados del Programa RenovAr. Rondas 1 y 1.5. MINEM. Recuperado de <www.minem.gob.ar/www/833/25897/proyectosadjudicados-del-programa-renovar $>$

Ministério de Minas e Energia (2015), Plano Decenal de Expansão de Energia 2024. Ministério de Minas e Energia-Empresa de Pesquisa Energética, Brasília, MME/EPE.

Ministerio de Producción (2017), Acuerdo por la Producción y el Empleo Principales lineamientos para el sector de Energías Renovables. Plan de consolidación e integración. Recuperado de <http://www.uia.org.ar/ download.do?id=6707>

Organización Internacional del Trabajo (огт) (2011), Skills and Occupational Needs in Renewable Energy. Ginebra, отт. Disponible en <www.ilo.org/ wcmsp5/groups/public/---ed_emp/---ifp_skills/documents/publication/ wcms_166823.pdf>

Pahle, M., Pachauri, S. y Steinbacher, K. (2016), "Can the Green Economy Deliver it All? Experiences of Renewable Energy Policies with Socio-economic Objectives", Applied Energy, 179. DOI <https://doi.org/10.1016/j. apenergy.2016.06.073> 
Pérez, C., Marín, A. y Navas-Aleman, L. (2014), "The Possible Dynamic Role of Natural Resource-based Networks in Latin American Development Strategies", en G. Dutrénit y J. Sutz (eds.), Innovation Systems for Inclusive Development: The Latin American Experience, Cheltenham, Edward Elgar.

Porcile, G. y Martins, A. (2017), "Cambio estructural, crecimiento y política industrial”, en M. Cimoli (ed.), Politicas industriales y tecnológicas en América Latina, Santiago, CePAL-BUz/GIz.

Puerto Rico, J. y Sauer, I.L. (2015), "Política energética en Brasil: implementación del componente renovable", Energética, 45. Disponible en $<$ https:// revistas.unal.edu.co/index.php/energetica/article/download/47366/pdf>.

Recalde, M., Bouille, D. y Girardin, L. (2015), "Limitaciones para el desarrollo de energías renovables en Argentina", Problemas del Desarrollo. Revista Latinoamericana de Economía, vol. 46, núm. 183, México, IIEc-unam. Disponible en <http://www.scielo.org.mx/scielo.php?pid=S03017036201 5000400089\&script=sci_arttext\&tlng=pt $>$

Resolución SE 108/2011, Habilitase la realización de Contratos de Abastecimiento entre el Mercado Eléctrico Mayorista y las ofertas de disponibilidad de generación y energía asociada. Boletín Oficial de la República Argentina, Buenos Aires (13 de abril de 2011).

Rogge, K.S., Kern, F. y Howlett, M. (2017), "Conceptual and Empirical Advances in Analysing Policy Mixes for Energy Transitions", Energy Research y Social Science, 33. DOI <https://doi.org/10.1016/j.erss.2017.09.025>

Secretaría de Energía (sENer) (2017), Reporte de Avance de Energías Limpias 2017, México.

Siboldi, A. (2016), "Aspectos institucionales, legales, regulatorios. Marco normativo de las energías renovables. Agencia de ERNC y ahorro de energía”, en L.M. Rotaeche y G.A. Rabinovich (eds.), Energías renovables no convencionales: Argentina frente al desafio de un futuro sostenible, Buenos Aires, Instituto Argentino de la Energía "General Mosconi".

Sica, D. (2016), "El desarrollo de la industria y la integración de las energías renovables", en L.M. Rotaeche y G.A. Rabinovich (eds.), Energias renovables no convencionales: Argentina frente al desafío de un futuro sostenible, Buenos Aires, Instituto Argentino de la Energía "General Mosconi".

Silva Colomer, J., Blanco Silva, F., Maroto, C., Donato, L. y López Díaz, A. (2014), "Análisis de las energías renovables en producción eléctrica: Estudio comparativo entre España y Argentina", Revista Peruana de Energía, núm. 4. Disponible en <http://www.santivanez.com.pe/wp-content/ uploads/2015/05/2-Analisis-Energias-Renovables.pdf> 
Subsecretaría de Energías Renovables (2016), Energías Renovables en Argentina. Oportunidades, Desafios, Acciones, Buenos Aires, Subsecretaría de Energías Renovables. Disponible en <www.energiaestrategica.com/wp-content/ uploads/2017/08/20170809_Viento-y-Energia_UBA_SKind.pdf>

Unruh, G. (2000), "Understanding Carbon Lock-in”, Energy Policy, 28(12). DOI <https://doi.org/10.1016/S0301-4215(00)00070-7> (2002), "Escaping Carbon Lock-in", Energy Policy, 30(4). DOI <https://doi.org/10.1016/S0301-4215(01)00098-2>

Weiss, I., Sprau, P. y Helm, P. (2003), “The German pv Solar Power financing Schemes reflected on the German pv Market", Proc. $3^{\text {rd }}$ World Conference on Photovoltaic Energy Conversion, Osaka.

World Energy Council (2017), Escenarios energéticos mundiales, Londres, World Energy Council.

Yoguel, G., Barletta, F. y Pereira, M. (2017), "Los aportes de tres corrientes evolucionistas neoschumpterianas a la discusión sobre políticas de innovación”, Revista Brasileira de Inovação, 16(2). DOI <https://doi.org/10.20396/ rbi.v16i2.8650116>

Zhang, S., Andrews-Speed, P., Zhao, X. y He, Y. (2013), "Interactions between Renewable Energy Policy and Renewable Energy Industrial Policy: A Critical Analysis of China's Policy Approach to Renewable Energies", Energy Policy, 62. DOI <https://doi.org/10.1016/j.enpol.2013.07.063> 